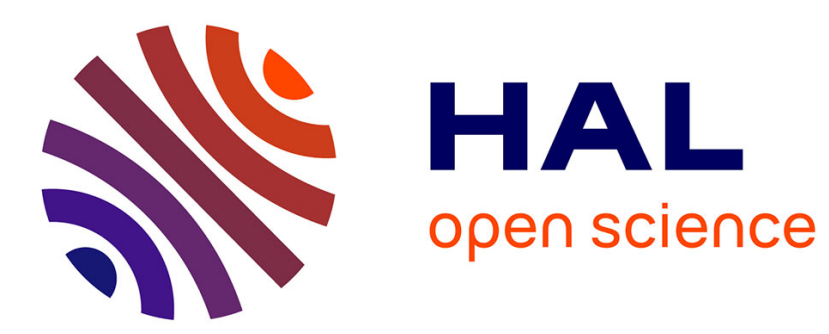

\title{
Pricing bivariate option under GARCH processes with time-varying copula
}

Jing Zhang, Dominique Guegan

\section{To cite this version:}

Jing Zhang, Dominique Guegan. Pricing bivariate option under GARCH processes with time-varying copula. Insurance: Mathematics and Economics, 2008, 42 (3), pp.1095-1103. 10.1016/j.insmatheco.2008.02.003 . halshs-00286054

\section{HAL Id: halshs-00286054 \\ https://shs.hal.science/halshs-00286054}

Submitted on 15 Apr 2009

HAL is a multi-disciplinary open access archive for the deposit and dissemination of scientific research documents, whether they are published or not. The documents may come from teaching and research institutions in France or abroad, or from public or private research centers.
L'archive ouverte pluridisciplinaire HAL, est destinée au dépôt et à la diffusion de documents scientifiques de niveau recherche, publiés ou non, émanant des établissements d'enseignement et de recherche français ou étrangers, des laboratoires publics ou privés. 


\title{
Pricing bivariate option under GARCH processes with time-varying copula
}

\author{
J. Zhang a,b,c,*, D. Guégan ${ }^{\mathrm{c}}$

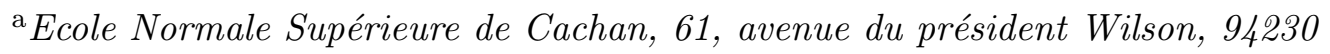 \\ Cachan Cedex, France \\ ${ }^{\mathrm{b}}$ East China Normal University, Department of Statistics, 3663 Zhongshan North \\ Road, 200063 Shanghai, China \\ ${ }^{\mathrm{c}}$ Université Paris 1 Panthéon-Sorbonne, CES - MSE, 106 Boulevard de l'Hopital, \\ 75013 Paris, France.
}

\begin{abstract}
This paper develops a method for pricing bivariate contingent claims under General Autoregressive Conditionally Heteroskedastic (GARCH) process. As the association between the underlying assets may vary over time, the dynamic copula with time-varying parameter offers a better alternative to any static model for dependence structure and even to the dynamic copula model determined by dynamic dependence measure. Therefore, the proposed method proves to play an important role in pricing bivariate options. The approach is illustrated with one type of better-of-two-markets claims: call option on the better performer of Shanghai and Shenzhen Stock Composite Indexes. Results show that the option prices obtained by the time-varying copula model differ substantially from the prices implied by the static copula model and even the dynamic copula model derived from the dynamic dependence measure. Moreover, the empirical work displays the advantages of the suggested method.
\end{abstract}

Key words: call-on-max option, GARCH process, Kendall's tau, Copula, Dynamic Copula, time-varying parameter

JEL: C02, C32, G13

Subject Category and Insurance Branch Category: IE50

* Corresponding author.

Email address: celine_chang_12@hotmail.com (J. Zhang). 


\section{Introduction}

Following the great work of Black and Scholes (1973) and Merton (1973), the option literature has been developed as an important research field. Over the years, a lot of pricing models have been put forward. Considering the heteroskedasticity of assets, Duan (1995) explored the extension of the risk neutralization in Rubinstein (1976) and Brennan (1979). In Duan (1995), options were priced when the dynamics of the underlying asset price followed a GARCH process through an equilibrium argument, which provided a relatively easy transformation to risk-neutral environment.

As multivariate options are regarded as excellent tool for hedging risk in finance, the dependence structure plays an important role. Various models in this aspect, Margrabe (1978), Stulz (1982), Johnson (1987), Reiner (1992) and Shimko (1994), for instance, applied the correlation to measure the dependence between the assets. However, as Embrechts et al. (2002) and Forbes and Rigobon (2002) have pointed out, correlation may cause some confusion and misunderstanding. Indeed, it is a financial stylized fact that correlations observed under ordinary market conditions differ substantially from those observed in hectic periods. Therefore, a more appropriate measure for the dependence structure is called for. One of the ideal measures is the copula, as it contains all the information about the dependence structure and captures nonlinear dependence. Copulas have been introduced to price bivariate options in Rosenberg (1999) and Cherubini and Luciano (2002). In these papers, copulas were applied in a static way. However, most of financial data often cover a reasonably long time period, so the economic factors may induce some changes in the dependence structure. Therefore, to price the bivariate option more reasonably, a dynamic copula approach should be adopted.

Recently, Goorbergh et al. (2005) applied the dynamic copula models to price the bivariate option under GARCH process, this method is referred to hereinafter as "Goorbergh's method" for convenience. Goorbergh's method describes the dynamics of copula by allowing the dependence parameter (specifically say, Kendall's tau) to evolve according to a particular regression equation. The forcing variables in this equation are the conditional volatilities of the underlying assets. With this regression equation, dynamic Kendall's tau is computed and further is used to decide the parameters of several one-parameter copulas. It should be noted that in Goorbergh's method, the dynamics is only reflected in the rolling-window and time series regression. Moreover, there are three aspects calling for attention: (1) The size of the rolling-window may influence the result for the option prices; (2) The specified time series regression equation may fail to represent the dynamic dependence correctly; (30 The copula families are restricted to one-parameter ones. 
In the present paper, a new dynamic approach to price bivariate options with GARCH process and time-varying copula is proposed. For the innovations obtained from GARCH filtering of the underlying assets, a series of the best copulas are selected for different subsamples divided by moving windows under AIC criterion (Akaike, 1974). Through this process, the copula changes can be observed clearly, which further works for specifying the dynamics in the dependence structure. An innovative feature of the present paper is investigating the dynamic evolution of the copula's parameter as a time-varying function of some predetermined variables, which gives a tractable dynamic expression to the changes of the copula. In this proposed approach, copulas are not limited to one-parameter family, multi-parameter copulas can also be considered. Compared with the previous methods, the approach in the present paper makes the dynamic pricing more reasonable and tractable.

The approach is illustrated with the call option on the better performer based on two important Chinese stock indexes, Shanghai Stock Composite Index and Shenzhen Stock Composite Index. Results show that the option prices implied by time-varying copula differ substantially from the prices obtained in the case of static dependence structure. Moreover, significant differences are also observed between the option prices implied by two dynamic copula methods: the proposed method and Goorbergh's method.

The remainder of this paper is organized as follows. In Section 2, the preliminary knowledge is reviewed and some related work is introduced. Section 3 explains in detail the new idea for pricing bivariate option under GARCH process with time-varying copula. In section 4, empirical study is described and results are presented. Section 5 concludes.

\section{Preliminaries and Related Work}

\subsection{Option valuation}

This paper concentrates on European option on the better performer of two assets, but the technique is sufficiently general to apply for other alternative multivariate options as well. The call option on the better performer can be referred to as call-on-max option. The payoff of a unit amount call-on-max option would be

$$
\max \left\{\max \left(S_{1, T}, S_{2, T}\right)-K, 0\right\}
$$

where $S_{i, t}$ is the price at time $t$ of the $i$-th asset $(i=1,2), T$ denotes the maturity, and $K$ is the strike price. In the following, $r_{i, t}$ is used to denote the log-return on $i$-th index $(i=1,2)$ from time $t-1$ to time $t$, i.e., $r_{i, t}=\log \frac{S_{i, t}}{S_{i, t-1}}$, and the corresponding exponential expression is $R_{i, t}=\exp \left(r_{i, t}\right)$. 
The fair value of the option is obtained by taking the discounted expected value of the option's payoff under the risk-neutral probability measure. As the call-on-max option is typically traded over the counter, price data are not available. Therefore, valuation models cannot be tested empirically. However, comparing models with different assumptions can be implemented.

\subsection{Risk neutralization with GARCH process}

In order to derive the joint risk-neutral return process from the objective bivariate distribution, the objective marginals are specified. Instead of deriving the bivariate risk-neutral distribution directly, each marginal process is proposed to transform separately. The one-period log-return for every index is assumed to be conditionally distributed under the objective probability measure $P$, together with a GARCH process (Bollerslev, 1986), that is, for $i=1,2$ :

$$
\begin{gathered}
r_{i, t}=\mu_{i}+\varepsilon_{i, t}, \\
h_{i, t}=\alpha_{i, 0}+\sum_{j=1}^{q} \alpha_{i, j} \varepsilon_{i, t-j}^{2}+\sum_{j=1}^{p} \beta_{i, j} h_{i, t-j}, \\
\varepsilon_{i, t} \mid \varphi_{i, t-1} \sim N\left(0, h_{i, t}\right) \quad \text { under measure P, }
\end{gathered}
$$

where $\mu_{i}$ is the drift, the set $\varphi_{i, t-1}$ corresponds to the information set $(\sigma$ field) of all information up to and including time $t-1$. Other restrictions are $p, q \in \mathbb{N} ; \alpha_{i, 0}>0 ; \alpha_{i, j} \geq 0(j=1, \ldots, q) ; \beta_{i, j} \geq 0(j=1, \ldots, p)$. To ensure covariance stationarity of the $\operatorname{GARCH}(p, q)$ process, $\sum_{j=1}^{q} \alpha_{i, j}+\sum_{j=1}^{p} \beta_{i, j}$ is assumed to be less than 1 .

In order to obtain the risk-neutral price, the conventional risk-neutral valuation relationship should be generalized to accommodate the heteroskedasticity of the asset return process, thus a risk-neutral probability measure $Q$ is derived:

Assumption 1 A pricing measure $Q$ is said to satisfy the locally risk-neutral valuation relationship if the following conditions are all satisfied:

(1) measure $Q$ is mutually absolutely continuous with respect to the objective measure $P$;

(2) $r_{i, t} \mid \varphi_{i, t-1}$ normally distributes under measure $Q$;

(3) $E^{Q}\left(R_{i, t} \mid \varphi_{i, t-1}\right)=e^{r_{f}}$, where $r_{f}$ denotes the constant risk-free interest rate;

(4) $\operatorname{Var}^{Q}\left(r_{i, t} \mid \varphi_{i, t-1}\right)=\operatorname{Var}^{P}\left(r_{i, t} \mid \varphi_{i, t-1}\right)$ almost surely with respect to the objective measure $P$.

Under some conditions, the locally risk-neutral valuation relationship holds (Duan, 1995), and the log-return processes in Equation (1) can be transformed into the one under the risk-neutral environment: 
Theorem 2 Under the locally risk-neutral probability measure $Q$, the model for one-period log-return $r_{i, t}$ becomes

$$
\begin{gathered}
r_{i, t}=r_{f}-\frac{1}{2} h_{i, t}+\xi_{i, t}, \\
h_{i, t}=\alpha_{i, 0}+\sum_{j=1}^{q} \alpha_{i, j}\left(r_{i, t-j}-\mu_{i}\right)^{2}+\sum_{j=1}^{p} \beta_{i, j} h_{i, t-j}, \\
\xi_{i, t} \mid \varphi_{i, t-1} \sim N\left(0, h_{i, t}\right) \quad \text { under measure } Q .
\end{gathered}
$$

Proof is presented in Appendix.

Theorem 2 provides a relatively easy transformation from the objective model to the risk-neutral one. According to this theorem, the terminal asset price is derived in the following corollary:

Corollary 3 When the locally risk-neutral valuation relationship holds, the terminal price for the $i$-th $(i=1,2)$ asset can be expressed as:

$$
S_{i, T}=S_{i, t} \exp \left[(T-t) r_{f}-\frac{1}{2} \sum_{s=t+1}^{T} h_{i, s}+\sum_{s=t+1}^{T} \xi_{i, s}\right] .
$$

For the importance of the martingale property for the theory of contingent claim pricing, it is necessary to note that the discount asset price process $e^{-r_{f} t} S_{i, t}$ is a $Q$-martingale.

Therefore, under the locally risk-neutral probability measure $Q$, the call-onmax option with exercise price $K$ maturing at time $T$ has the $t$-time value:

$$
C O M_{t}=e^{-(T-t) r_{f}} E^{Q}\left[\max \left(\max \left(S_{1, T}, S_{2, T}\right)-K, 0\right)\right]
$$

\subsection{Conditional copula}

In order to price the bivariate option in a dynamic way, the concept of conditional copula is applied. Patton (2006) has introduced the conditional copulas, which are associated with conditional laws in a particular way. And Sklar's theorem (Sklar , 1959) has been extended for conditional distributions and conditional copulas:

Theorem 4 Let $F$ be a d-dimensional conditional distribution function with continuous margins $F_{1}, F_{2}, \cdots, F_{d}$, and let $\mathcal{F}$ be some conditioning set, then there exists a unique conditional d-copula $C:[0,1]^{d} \rightarrow[0,1]$ such that for all $\mathbf{x}$ in $\overline{\mathbb{R}}^{d}$,

$$
F\left(x_{1}, x_{2}, \cdots, x_{d} \mid \mathcal{F}\right)=C\left(F_{1}\left(x_{1} \mid \mathcal{F}\right), F_{2}\left(x_{2} \mid \mathcal{F}\right), \cdots, F_{d}\left(x_{d} \mid \mathcal{F}\right)\right) .
$$


Conversely, if $C$ is a conditional d-copula and $F_{1}, F_{2}, \cdots, F_{d}$ are conditional distribution functions, then the function $F$ defined by Equation (5) is a ddimensional conditional distribution function with margins $F_{1}, F_{2}, \cdots, F_{d}$.

We can see from the above properties that a conditional copula is exactly the copula concept in the conditional environment.

\section{Option pricing under GARCH process with time-varying copula: Methodology}

Instead of transforming the joint distribution into its risk-neutral counterpart as a whole, the objective marginals specified in Equation (1) are transformed into the locally risk-neutral forms in Equation (2) respectively, and the objective joint distribution is then transformed into the risk-neutral one through copulas. In the proposed scheme for valuating the bivariate option, the objective bivariate distribution of the log-returns $\left(r_{1, t}, r_{2, t}\right)$ is specified conditionally on $\varphi_{t-1}=\sigma\left(\left(r_{1, s}, r_{2, s}\right): s \leq t-1\right)$. A restriction is set that all the conditional margins only depend on their own past, i.e., $\varphi_{t-1}=\left(\varphi_{1, t-1}, \varphi_{2, t-1}\right)$, where $\varphi_{i, t-1}=\sigma\left(r_{i, s}: s \leq t-1\right)$ denotes the conditional information on $i$-th variable available at time $t-1$. Therefore, the proposed technique is that the objective copula and the risk-neutral copula are assumed to be the same. For the conditional copula, not only one-parameter copulas can be used, choices are enriched to consider also two-parameter copulas such as Student $t$ copula and convex combination Archimedean copulas. With the aim of choosing the best fitting copula, AIC criterion (Akaike, 1974) is used.

Over the whole period, one best copula is firstly chosen to fit the log-return innovations in Equation (1). Then using moving windows helps to observe the change of the copula and further to work out a reasonable dynamic method. On different subsamples divided by the moving windows, the best copulas are selected according to AIC criterion. If the results show that the copula family remains changeless while the copula parameters change, the innovative method is to define a time-varying parameter function. Such dynamic function is of the GARCH type structure, that is, the dynamic copula $C$ for $\left(\varepsilon_{1, t}, \varepsilon_{2, t}\right)$ in Equation (1) is assumed to have the time dependent parameter vector $\theta_{t}=\left(\theta_{1, t}, \theta_{1, t}, \ldots, \theta_{m, t}\right)$, such that

$$
\theta_{l, t}=\theta_{0}+\sum_{i=1}^{g} \eta_{i} \prod_{j=1}^{2} \varepsilon_{j, t-i}+\sum_{k=1}^{s} \zeta_{k} \theta_{l, t-k}
$$

for $l=1,2, \ldots, m$ and $\eta_{i}(i=1,2, \ldots, g), \zeta_{k}(k=1,2, \ldots, s)$ are scalar parameters. 
To estimate the parameters in Equation (6), the maximum likelihood method is needed. As the standardized innovations are assumed to distribute conditionally normal, the bivariate conditional distribution function is such that

$$
F\left(\left(\varepsilon_{1, t}, \varepsilon_{2, t}\right) ; \theta_{t}\right)=C\left(\Phi\left(\varepsilon_{1, t}\right), \Phi\left(\varepsilon_{2, t}\right) ; \theta_{t}\right),
$$

where $C$ is the copula function, $\Phi$ is the marginal normal distribution function. The corresponding conditional density function is then

$$
f\left(\left(\varepsilon_{1, t}, \varepsilon_{2, t}\right) ; \theta_{t}\right)=c\left(\Phi\left(\varepsilon_{1, t}\right), \Phi\left(\varepsilon_{2, t}\right) ; \theta_{t}\right) \prod_{i=1}^{2} \phi\left(\varepsilon_{i, t}\right),
$$

where the copula density $c$ is given by

$$
c\left(u_{1}, u_{2} ; \theta\right)=\frac{\partial^{2} C\left(u_{1}, u_{2} ; \theta\right)}{\partial u_{1} \partial u_{2}},
$$

with $\left(u_{1}, u_{2}\right) \in[0,1]^{2}$ and $\phi$ represents the normal density function. Therefore, the conditional log-likelihood function can be finally evaluated as

$$
\sum_{t=b+1}^{n}\left(\log c\left(\Phi\left(\varepsilon_{1, t}\right), \Phi\left(\varepsilon_{1, t}\right) ; \theta_{t}\right)+\sum_{i=1}^{2} \log \phi\left(\varepsilon_{i, t}\right)\right)
$$

where $b=\max (p, r)$.

Specifically, for one-parameter copulas, the time-varying parameter function can be presented directly, but for two-parameter copulas, the complexity of estimating parameters results in choosing one to change while the other one being static.

For instance, for the dynamic Student $t$ copula, if the parameter of degrees of freedom $\nu$ is assigned to remain static, the dynamic correlation can be defined as:

$$
\rho_{t}=h^{-1}\left(r_{0}+r_{1} \varepsilon_{1, t-1} \varepsilon_{2, t-1}+r_{2} h\left(\rho_{t-1}\right)\right),
$$

where $r_{0}, r_{1}, r_{2}$ are parameters and $h(\cdot)$ is Fisher's transformation for the correlation:

$$
h(\rho)=\log \left(\frac{1+\rho}{1-\rho}\right) .
$$

On the other hand, if the degrees of freedom $\nu$ change apparently along the time, while the variation of the correlation seems insignificant, then the correlation is regarded as static and $\nu$ is defined as dynamic parameter according to the time-varying function:

$$
\nu_{t}=l^{-1}\left(s_{0}+s_{1} \varepsilon_{1, t-1} \varepsilon_{2, t-1}+s_{1} l\left(\nu_{t-1}\right)\right),
$$


where $s_{0}, s_{1}, s_{2}$ are parameters and $l(\cdot)$ is a function defined as

$$
l(\nu)=\log \left(\frac{1}{\nu-2}\right)
$$

to ensure that the value of the degrees of freedom is not smaller than 2 .

As the objective and the locally risk-neutral conditional copulas are the same, pairs of standard normal random variables are drawn from the dynamic copula whose parameters are estimated by the above method under the objective probability measure. These random variables are regarded as the transformed innovations in the risk-neutral environment in Equation (2). This procedure is accomplished with the aid of Monte Carlo simulations. The generated random variables are then used to calculate the log-returns in Equation (2). Eventually, according to Corollary 3, the payoffs are averaged and discounted at the riskfree rate, and the fair value of the call-on-max option can be expressed as in Equation (4).

\section{Empirical work}

\subsection{Analysis of data set}

The valuation approach for the bivariate option under GARCH process with time-varying copula outlined in Section 3 is illustrated with call-on-max option on Shanghai and Shenzhen Stock Composite Indexes. The sample contains 1857 daily observations from January 4, 2000 to May 29, 2007.

The parameter estimates of GARCH $(1,1)$ processes (see Equation (1)) for the underlying assets' log-returns are listed in Table 1.

Table 1

Estimates of GARCH parameters for marginal log-return processes

\begin{tabular}{ccc}
\hline Parameter & Estimates for Shanghai index & Estimates for Shenzhen index \\
\hline$\mu$ & $3.833 \mathrm{e}-04(2.419 \mathrm{e}-04)$ & $3.761 \mathrm{e}-04(2.882 \mathrm{e}-04)$ \\
$\alpha_{0}$ & $5.136 \mathrm{e}-06(7.682 \mathrm{e}-07)$ & $5.529 \mathrm{e}-06(9.011 \mathrm{e}-07)$ \\
$\alpha_{1}$ & $8.115 \mathrm{e}-02(4.726 \mathrm{e}-03)$ & $8.721 \mathrm{e}-02(5.496 \mathrm{e}-03)$ \\
$\beta_{1}$ & $8.966 \mathrm{e}-01(5.034 \mathrm{e}-03)$ & $8.950 \mathrm{e}-01(5.249 \mathrm{e}-03)$ \\
AIC & -10793.11 & -10518.3 \\
\hline
\end{tabular}

Figures in brackets are standard errors.

The estimated standardized GARCH $(1,1)$ innovations are described in Figure 
1. It is noted that the outliers typically occur simultaneously and almost in the same direction.
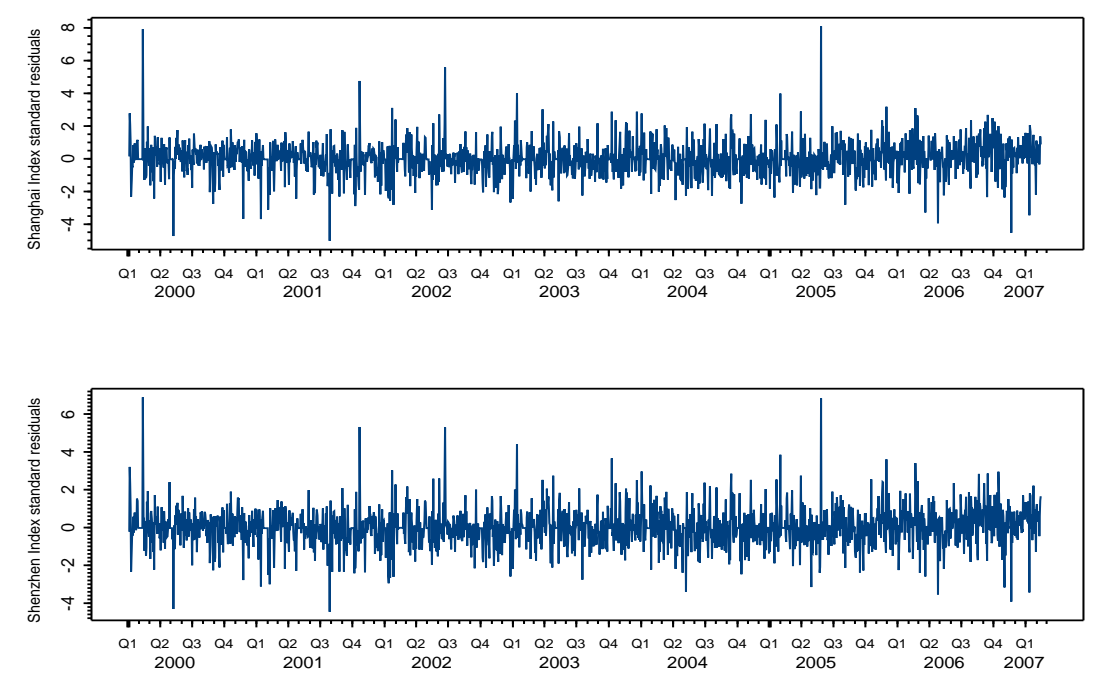

Fig. 1. Standardized residuals of GARCH $(1,1)$ for Shanghai and Shenzhen Stock Composite Indexes

\subsection{Dynamic copula method}

The scatter-plot of the support set of the empirical copula for the standardized log-return innovations is shown in Figure 2, which further proves the positive dependence between the two series. According to the scatter-plot, several kinds of copulas are considered to describe the dependence structure, including Gaussian, Student $t$, Frank, Gumbel, Clayton copulas, etc. For details on these copulas, see Joe (1997) and Nelsen (1999).

All the copulas mentioned above are fitted to the support set of the standardized innovation pairs. Copula fitting result is listed in Table 2, where AIC criterion is used to choose the best fitting copula.

From the fitting result, the one which has the smallest AIC value is the Student $t$ copula. Therefore, Student $t$ copula with degrees of freedom $\nu=5.807$ and correlation $\rho=0.9349$ is considered as the best fitting copula in the case of static dependence structure.

For the dynamics in the dependence structure, Goorbergh's method assumes that the dependence parameter evolves according to a particular time series regression equation. This method is displayed here in order to compare with the proposed method in the present paper. Specifically, let $\tau_{t}$ be Kendall's tau 


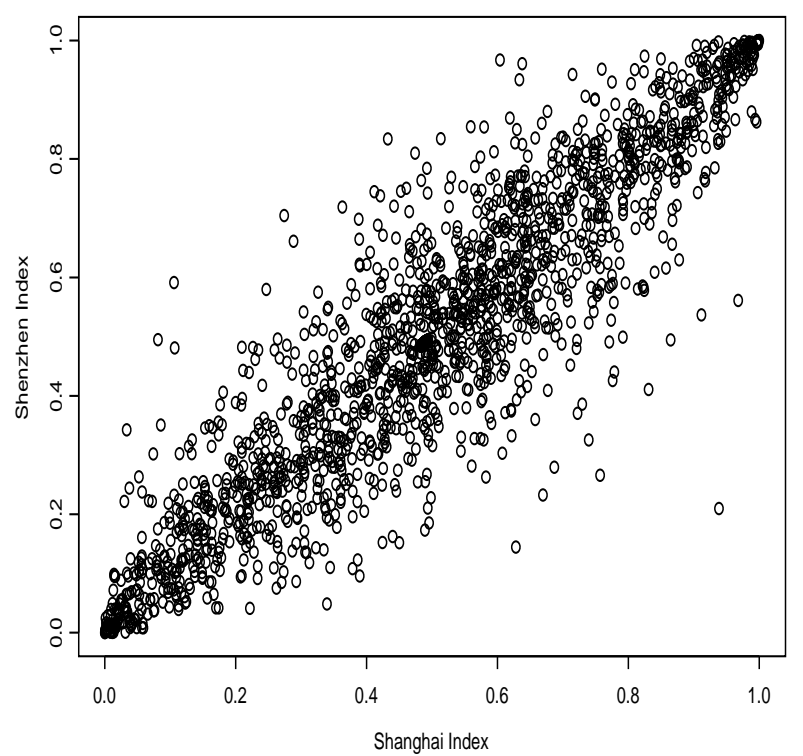

Fig. 2. Scatter plot of the support set for Shanghai and Shenzhen standard residuals Table 2

Copula Fitting Results

\begin{tabular}{ccc}
\hline Copula & Parameter & AIC value \\
\hline Student $t$ & $9.349 \mathrm{e}-01(5.095 \mathrm{e}-02) ;$ & -3797.926 \\
& $5.807(8.078 \mathrm{e}-01)$ & \\
Gaussian & $9.314 \mathrm{e}-01(4.402 \mathrm{e}-02)$ & -3757.412 \\
Frank & $16.593(3.611 \mathrm{e}-01)$ & -3591.436 \\
Gumbel & $3.971(7.845 \mathrm{e}-02)$ & -3528.21 \\
Clayton & $4.081(1.114 \mathrm{e}-01)$ & -2728.87 \\
\hline
\end{tabular}

Figures in brackets are standard errors and for Student $t$ copula, the first parameter is correlation and the second one is degree of freedom.

at time $t$, Goorbergh's method supposes that $\tau_{t}$ has a relationship with the conditional volatilities of the indexes, which is expressed as follows:

$$
\tau_{t}=\gamma_{0}+\gamma_{1} \log \max \left(h_{1, t}, h_{2, t}\right)
$$

Using the rolling window with the width of 20 days, the Kendall's tau $\tau_{t}$ is calculated, thus the parameters $\gamma_{0}$ and $\gamma_{1}$ are estimated by regressing these Kendall's tau values on $\log \max \left(h_{1, t}, h_{2, t}\right)$ for all $t$. Sequently, the estimated Kendall's tau $\hat{\tau}\left(h_{1, t}, h_{2, t}\right)$ computed by Equation (9) is used to fix the conditional copula at time $t$, using the one-to-one relationship between Kendall's tau and the copula's parameter. This dynamic scheme leads to the restriction 
that only one-parameter copulas can be tractably considered. Some results for this method are shown in Figure 3, where the coefficient $\gamma_{0}$ is estimated at 0.9447 with standard error 0.0421 , and $\gamma_{1}$ is estimated at 0.0228 with standard error 0.0049 .
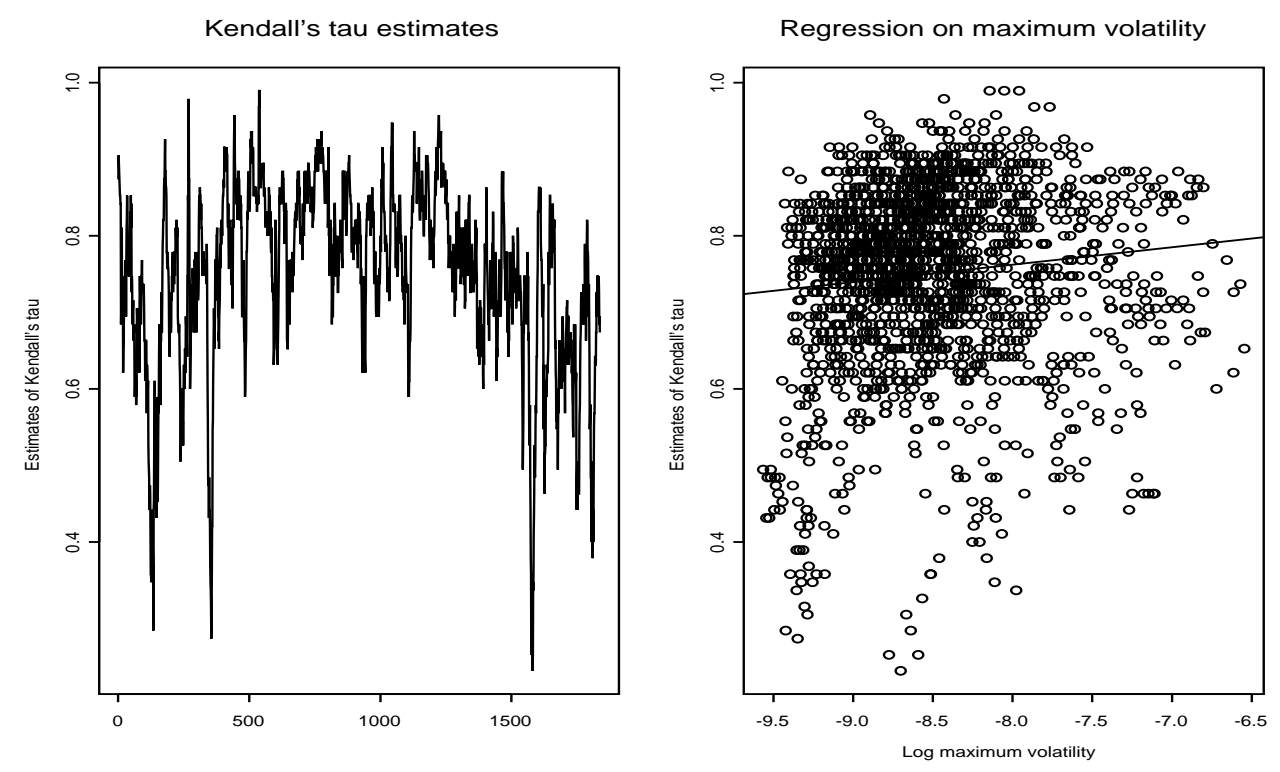

Fig. 3. Dynamic Kendall's tau estimated with rolling-window and its regression on logarithm of maximum volatilities in Goorbergh's method

The regression can somewhat describe the variation of the dependence, based on the assumption that the volatilities in both markets are highly dependent, hence such specification may influence the accuracy for the option pricing. Therefore, a more systematic and reasonable dynamic method is called for.

Using moving window allows to observe the changes of the copula in a direct way, and makes the dynamic specification more suitable to the real situation. The whole sample is divided into subsamples by 16 windows, each window consists of 300 observations and is moved per 100 observations. Along with the window's moving, series of best fitting copulas on the corresponding subsamples are selected by AIC criterion. The result is shown in Table 3, where not only the best copulas are presented, but also the second best copulas are listed with the aim of comparison.

The result listed in Table 3 shows that on almost all subsamples, Student $t$ copula exhibits it's well fitting: even though Student $t$ copulas are not the best fitting copulas for some windows, they prove to be the second best fitting ones within these windows with AIC values very close to the corresponding best fitting copulas. Therefore, it is rather reasonable to assume that the copula family remains static as Student $t$. In order to determine which parameter should be appropriately chosen as the dynamic one, two models specified in 
Equation (7) and Equation (8) are both considered.

If the correlation works as the dynamic parameter while the degrees of freedom being static, the estimates for the dynamic Student $t$ copula in Equation (7) cannot be achieved under convergence. And it is also obvious from Table 3 that for the best fitting Student $t$ copulas along with the moving window, the correlation changes from $8.765 \mathrm{e}-01$ to $9.730 \mathrm{e}-01$ with the change rate of $11.010 \%$, while the degrees of freedom change from 4.743 to 83.968 with the change rate of $1670.356 \%$. Therefore, it seems more reasonable to assume that the degrees of freedom vary with time while the correlation remains static. The fitting result for the dynamic copula with time-varying degrees of freedom defined in Equation (8) is presented in Table 4, with the corresponding parameter estimates and the AIC value of the static Student $t$ copula for comparison.

From Table 4, it can be observed according to the AIC value that the dynamic student $t$ copula model works better than the static one for the data.

\subsection{Bivariate option pricing}

Uniform distributed random variables are then generated from the dynamic Student $t$ copula and then are used to compute the bivariate option price. As the initial asset prices need to be close in order to let the option make sense, it is assumed here that they are normalized to unity, and the risk-free rate $r_{f}$ is assumed to be $4 \%$ per annum. The Monte Carlo study is based on 10,000 replications. Since the option price depends on the initial levels of the volatilities, the general initial volatility level and the high initial volatility level are both considered, where the general initial volatility is set according to $h_{i, 0}=\alpha_{i, 0} /\left(1-\alpha_{i, 1}-\beta_{i, 1}\right)$, and the high initial volatility is defined as four times of the general one. Moreover, the maturity is assumed to be 1 month (20 trading days) and the strike price is set at levels between 0.98 and 1.02.

Three results for option pricing are illustrated here for comparison. The first result only concerns the static copulas; The second result comes from Goorbergh's method; The third result is derived from the new idea of dynamic copula with time-varying parameter.

The first result of option prices as the function of the strike with static copulas and different initial volatilities are shown in Figure 4.

From Figure 4, three conclusions can be summarized :

(1) The option prices obtained from the models with high initial volatilities are always higher than those obtained from the same models with general 


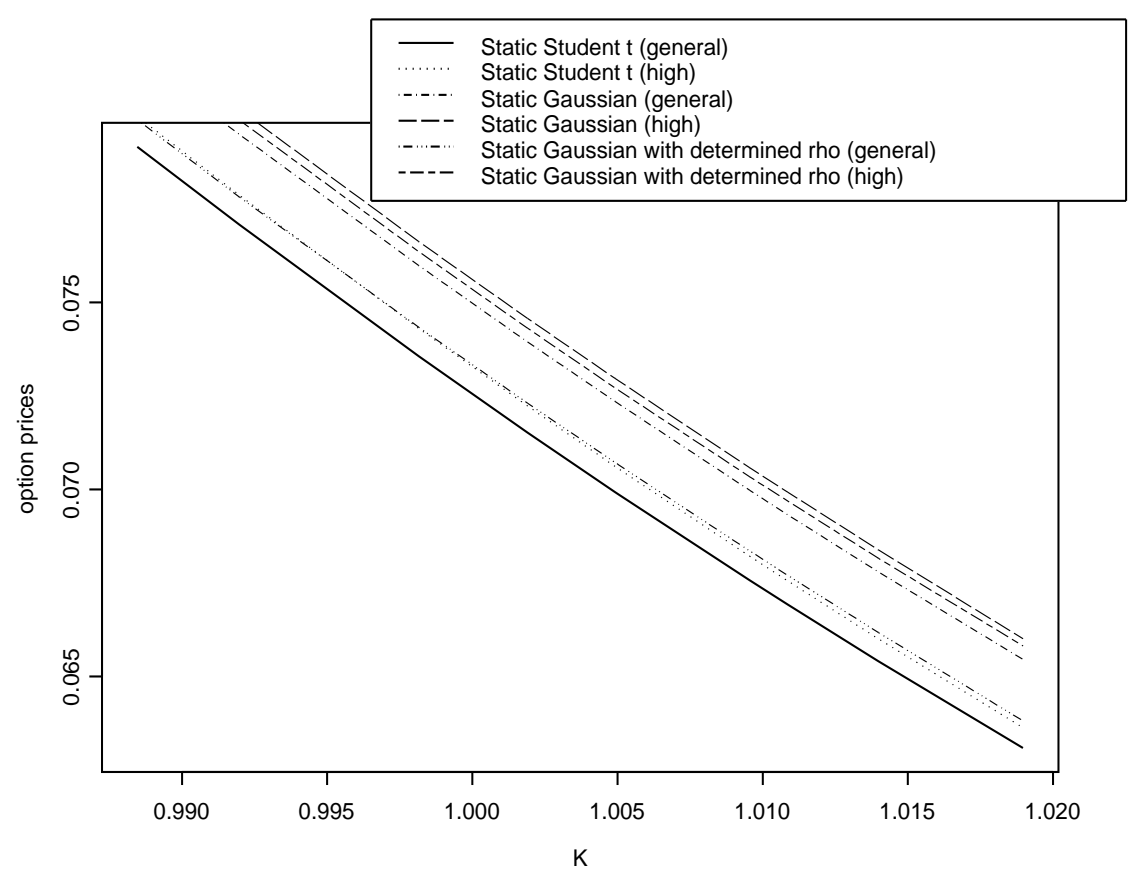

Fig. 4. Call-on-max option prices as a function of the strike using static copulas

"Static Student $t$ " and "Static Gaussian" represent the Student $t$ and Gaussian copulas chosen by AIC criterion over the whole sample; "general" and "high" mean that the GARCH model in Equation (1) has the general and high initial volatilities respectively; "Static Gaussian with determined rho" means that the correlation of the Gaussian copula is determined by Kendall's tau as in Goorbergh's method.

initial volatilities, which coincides with the financial general knowledge;

(2) The Gaussian copulas, whether chosen by AIC criterion or determined by Kendall's tau, always give the higher option prices than the student $t$ copula, and especially, the prices derived from the Gaussian copula chosen by AIC criterion win the highest, the prices derived from the Gaussian copula with correlation determined by Kendall's tau follow as the second highest, and the prices obtained from Student $t$ copula display the lowest;

(3) The influence of the initial volatility on the prices derived from the Gaussian copula with correlation determined by Kendall's tau shows the greatest, and the prices obtained from the Student $t$ and Gaussian copula chosen by AIC criterion are influenced moderately by the initial volatility.

The second result concerns Goorbergh's method, which is shown in Figure 5. Note that in Goorbergh's method, only the one-parameter copulas can be considered, therefore, only Gaussian copulas are displayed here.

From Figure 5, three conclusions are put forward:

(1) Again, the option prices obtained from the models with high initial volatilities are always higher than those obtained from the same models with 


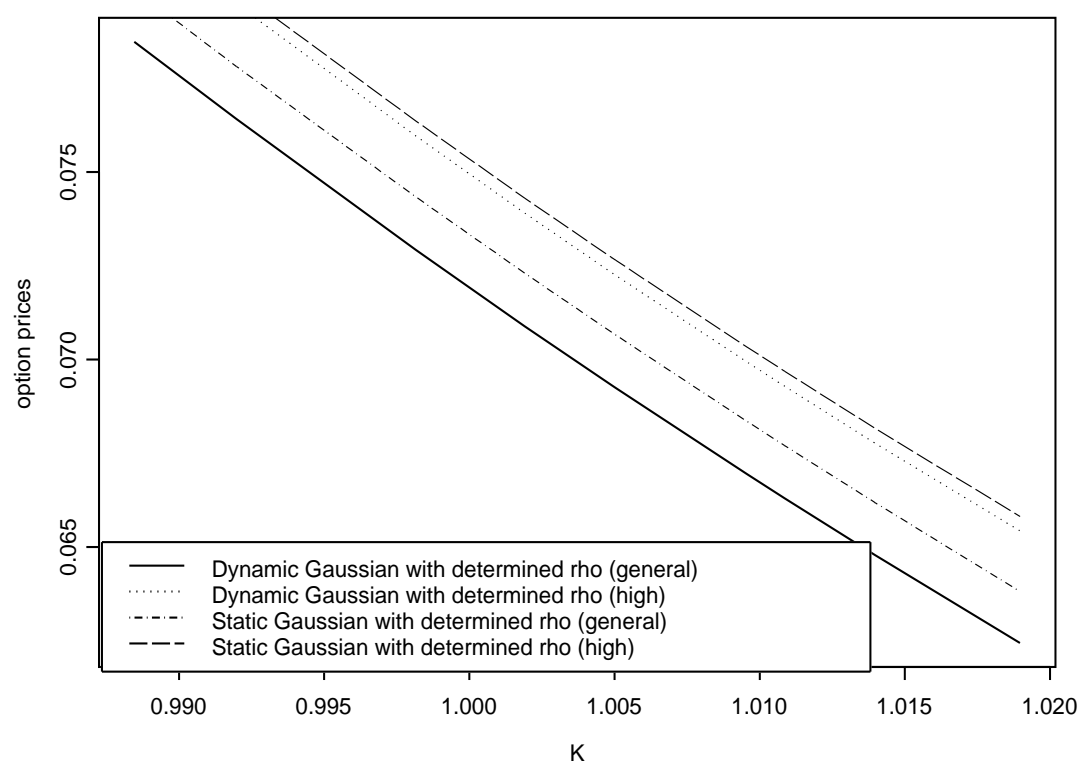

Fig. 5. Call-on-max option prices as a function of the strike in Goorbergh's method "Dynamic Gaussian with determined rho" and "Static Gaussian with determined rho" represent the Gaussian copulas with the dynamic and static correlation parameters determined by Kendall's tau respectively, as in Goorbergh's method; "general" and "high" mean that the GARCH model in Equation (1) has the general and high initial volatilities, respectively.

general initial volatilities;

(2) The static Gaussian copula model gives the higher option prices than the dynamic Gaussian copula model;

(3) The influence of the initial volatility on the dynamic Gaussian copula model is greater than that on the static one.

Using the proposed dynamic copula method, the third result is represented in Figure 6.

It can be concluded from Figure 6 that the option prices obtained from the models with high initial volatilities are always higher than those obtained from the same models with general initial volatilities. In order to clearly compare, the option prices obtained from the different models are presented in Figure 7, for the cases that the GARCH models in Equation (1) have the general and high initial volatilities, respectively.

From Figure 7, there come out the conclusions:

(1) In the general initial volatility case, the option prices derived from different models can be sorted the orders as follows: 


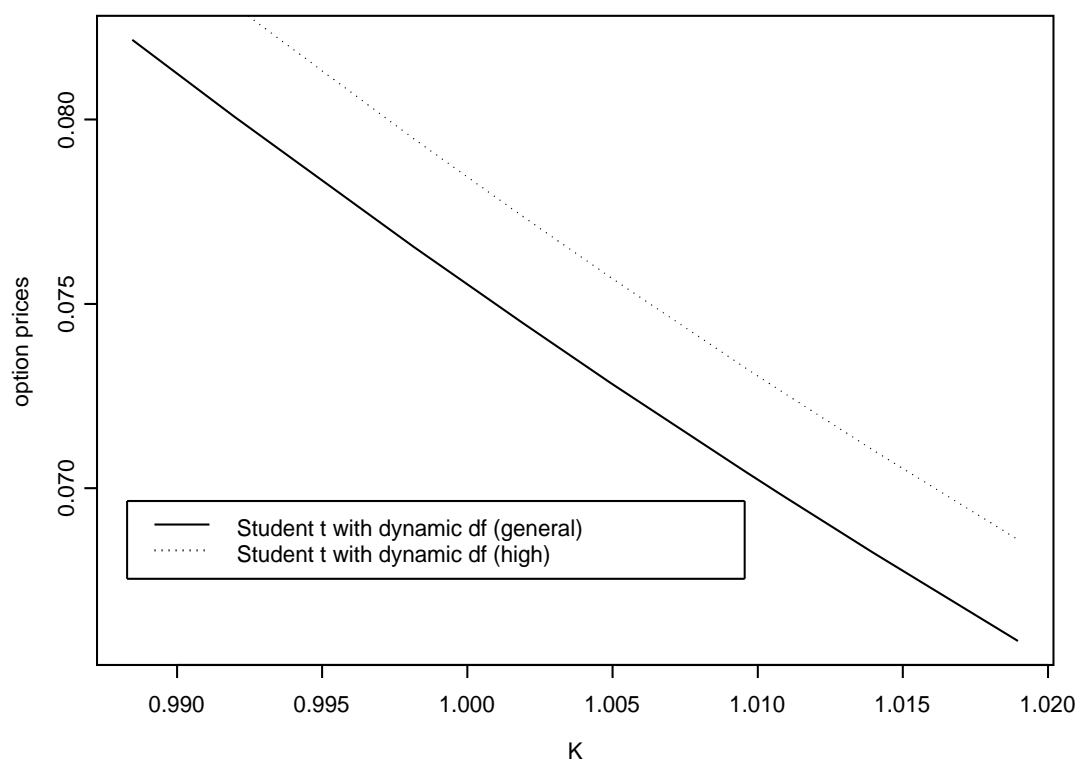

Fig. 6. Call-on-max option prices as a function of the strike using the dynamic Student $t$ copula with time-varying parameter

"Student $t$ with dynamic df" represents the dynamic Student $t$ copulas with timevarying degrees of freedom; "general" and "high" mean that the GARCH model in Equation (1) has the general and high initial volatilities, respectively.

Dynamic Gaussian copula with correlation determined by Kendall's tau $<$ Static Student $t$ copula $<$ Static Gaussian copula with correlation determined by Kendall's tau $<$ Static Gaussian copula $<$ Student $t$ copula with dynamic degrees of freedom;

(2) In the high initial volatility case, the option prices derived from different models can be sorted the orders as follows:

Static Student $t$ copula $<$ Dynamic Gaussian copula with correlation determined by Kendall's tau < Static Gaussian copula with correlation determined by Kendall's tau $<$ Static Gaussian copula $<$ Student $t$ copula with dynamic degrees of freedom;

(3) From the two conclusions above, the sort order changes only due to the model of dynamic Gaussian copula with correlation determined by Kendall's tau. In fact, from the plot, it can be observed that the influence of the initial volatility on the model of Gaussian copula with correlation determined by Kendall's tau does appear more marked than the other models, particularly for the model of Gaussian copula with dynamic correlation determined by Kendall's tau. The reason for this comes from Goorbergh's method where the dynamic Kendall's tau are obtained by the regression on the log-maximum of the volatilities. 

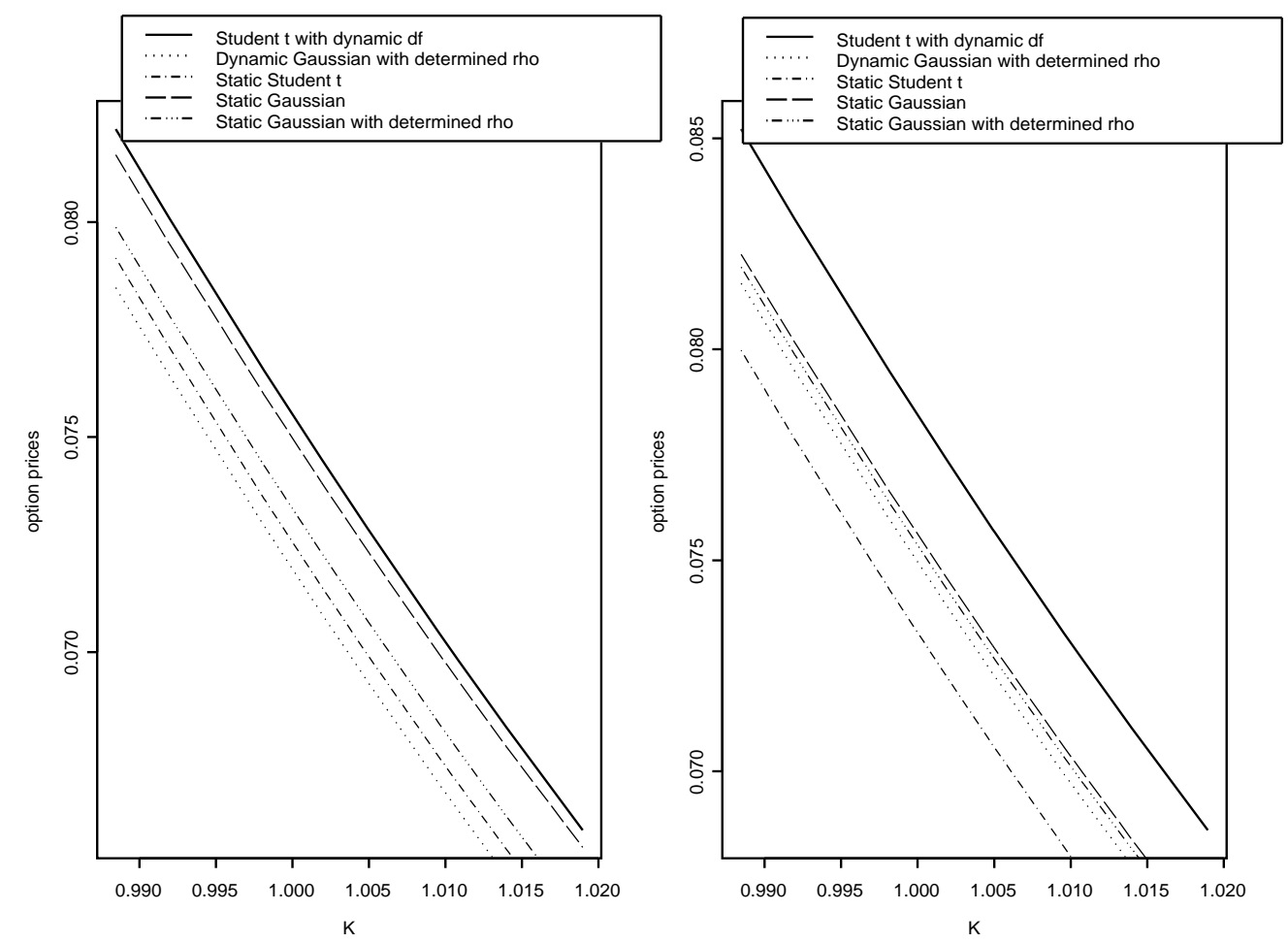

Fig. 7. Call-on-max option prices as a function of the strike using different models

"Student $t$ with dynamic df" represents the dynamic Student $t$ copulas with timevarying degrees of freedom; "Dynamic Gaussian with determined rho" and "Static Gaussian with determined rho" represent the Gaussian copulas with the dynamic and static correlation parameters determined by Kendall's tau respectively, as in Goorbergh's method; "Static Student $t$ " and "Static Gaussian" represent the Student $t$ and Gaussian copulas chosen by AIC criterion over the whole sample.

In conclusion, the sensitivity to the initial volatility of each model is somewhat manifested by the difference between the cases of general and high initial volatilities. One cannot deny the real influence of the initial volatility on the underlying assets and thus the option prices, but the specification of the dynamic Kendall's tau in Equation (9) may excessively specify such influence. After such specification, the copula determined by the Kendall's tau may be affected, and the sensitivity to the initial volatility may be not correctly reflected by the option prices.

\section{Conclusion}

In this paper, a systematic new approach for option pricing under GARCH process with time-varying copula model has been introduced, which provides some advantages. From the empirical result, the importance of choosing a 
"good" copula is affirmed, in both static and dynamic cases. Moreover, the limitations of Goorbergh's method are summarized. One is about the accuracy of the regression equation for estimating dynamic Kendall's tau. The second one comes from the specification on the volatility. The last one concerns the restriction of copula's family, that is, solely one-parameter copulas are applied. The contributions of the proposed method in the present paper can be generalized as follows: (1) Using the best copula makes the model more suitable; (2) For the dynamic case, observing the changes of the copula allows to more effectively create the dynamic model; (3) Dynamic copula with timevarying parameter is specified, which depicts the dynamics more reasonably and enables the changes to be more easily handled; (4) The dynamic method is not restricted to one-parameter copulas, multi-parameter copulas can also be used; (5) The influence of the volatility is not magnified excessively.

\section{A Proof of Theorem 2}

Since for $i=1,2, r_{i, t}\left|\varphi_{t-1}=\frac{S_{i, t}}{S_{i, t-1}}\right| \varphi_{t-1}$ normally distributes under measure $Q$, it can be written as

$$
\ln \frac{S_{i, t}}{S_{i, t-1}}=\omega_{i, t}+\xi_{i, t}
$$

where $\omega_{i, t}$ is the conditional mean and $\xi_{i, t}$ is a normal random variable with zero mean under the measure $Q$. And from the fourth condition of Assumption $1, h_{i, t}=\operatorname{Var}^{P}\left(r_{i, t} \mid \varphi_{t-1}\right)=\operatorname{Var}^{Q}\left(r_{i, t} \mid \varphi_{t-1}\right)$. Therefore, it can be obtained that

$$
E^{Q}\left(\frac{S_{i, t}}{S_{i, t-1}} \mid \varphi_{t-1}\right)=E^{Q}\left(e^{\omega_{i, t}+\xi_{i, t}} \mid \varphi_{t-1}\right)=e^{\omega_{i, t}+\frac{h_{i, t}}{2}}
$$

From the third condition of Assumption 1, $E^{Q}\left(\frac{S_{i, t}}{S_{i, t-1}} \mid \varphi_{t-1}\right)=e^{r_{f}}$, then it follows that

$$
\omega_{i, t}=r_{f}-\frac{1}{2} h_{i, t} .
$$

By the preceding result and Equation (1),

$$
\varepsilon_{i, t}=r_{i, t}-\mu_{i} .
$$

Substituting $\varepsilon_{i, t}$ into the conditional variance equation in Equation (1) yields the result.

\section{References}

Akaike, H., 1974. A new look to the statistical model identification. IEEE Transactions on Automatic Control, AC-19, 716-723. 
Black, F., Scholes, M.S., 1973. The pricing of options and corporate liabilities. J. Pol. Econ. 81, 637-654.

Bollerslev, T., 1986. Generalized autoregressive conditional heteroskedasticity. J. Econ. 81, 637-654.

Brennan, M., 1979. The pricing of contingent claims in discrete time models. J. Finance. 34, 53-68.

Cherubini, U., Luciano, E., 2002. Bivariate option pricing with copulas. Appl. Math. Finan. 9, 69-86.

Duan, J.-C., 1995. The GARCH option pricing model. Math. Finan. 5, 13-32.

Embrechts, P., McNeil, A., Strausmann, D., 2002. Correlation and dependence in risk management: properties and pitfalls. In: Dempster, M.A.H. (Ed.), Risk Management: Value at Risk and Beyond. Cambridge University Press, Cambridge, England 176-223.

Forbes, K., Rigobon, R., 2002. No contagion, only interdependence: Measuring stock market co-movements. J. Finance 57, 2223-2261.

Goorbergh, R. W.J. V. d., Genest, C., Werker, B.J.M., 2005. Bivariate option pricing using dynamic copula models. Insurance: Mathematics and Economics 37, 101-114.

Joe, H., 1997. Multivariae Models and Dependence Concepts. Chapman \& Hall, London.

Johnson, H., 1987. Options on the maximum or the minimum of several assets. J. Finan. Quant. Anal. 22, 277-283.

Margrabe, W., 1978. The value of an option to exchange one asset for another. J. Finan. 33, 177-186.

Merton, R., 1973. The theory of rational option pricing. Bell J. Econ. Management Sci. 4 141-183.

Nelsen, R., 1999. An introduction to copulas. Lecture Notes in Statistics No. 139. Springer, New York.

Patton, A. J., 2006. Modelling asymmetric exchange rate dependence. International Economic Review 47, 527-556.

Reiner, E., 1992. Quanto mechanics, From Black-Scholes to Black Holes: New Frontiers in Options. Risk Books, London 147-154.

Rosenberg, J.V., 1999. Semiparametric pricing of multivariate contingent claims. Working paper S-99-35, Stern School of Business, New York University, New York.

Rubinstein, M., 1976. The valuation of uncertain income streams and the pricing of options. Bell J. Econ. Management Sci. 7, 407-425.

Shimko, D.C., 1994. Options on futures spreads: Hedging, speculation, and valuation. J. Futures Markets 14, 183-213.

Sklar, A., Fonctions de répartition à n dimensions et leurs marges. Publications de l'Institut de Statistique de L'Université de Paris 8, 229-231.

Stulz, R.M., 1982. Options on the minimum or the maximum of two risky assets: analysis and applications. J. Finan. Econ. 10, 161-185. 
Table 3

Dynamic Copula Analysis using Moving Window

\begin{tabular}{|c|c|c|c|c|}
\hline$i$ & Best copula & $\mathrm{AIC}$ & Second best copula & $\mathrm{AIC}$ \\
\hline 1 & Ga: 9.308e-01 (1.033e-01) & -599.670 & $t: \begin{array}{c}9.282 \mathrm{e}-01(1.130 \mathrm{e}-01) \\
4.743(9.803 e-01)\end{array}$ & -584.889 \\
\hline 2 & Ga: 9.247e-01 (1.036e-01) & -486.515 & $t: \begin{array}{c}9.259 \mathrm{e}-01(8.959 \mathrm{e}-02) \\
20.022(1.239)\end{array}$ & -485.932 \\
\hline 3 & Ga: 9.381e-01 (1.103e-01) & -608.379 & $t: \begin{array}{c}9.384 \mathrm{e}-01(1.435 \mathrm{e}-01) ; \\
83.968(5.703 e)\end{array}$ & -606.581 \\
\hline 4 & $t: 9.539 \mathrm{e}-01(6.831 \mathrm{e}-02) ; 14.784(1.286)$ & -708.476 & Ga: $9.521 \mathrm{e}-01(1.247 \mathrm{e}-01)$ & -705.446 \\
\hline 5 & Ga: 9.646e-01 (1.437e-01) & -834.866 & $t: \begin{array}{c}9.656 \mathrm{e}-01(5.256 \mathrm{e}-01) ; \\
27.930(10.392)\end{array}$ & -834.836 \\
\hline 6 & $t: 9.730 \mathrm{e}-01(1.437 \mathrm{e}-01) ; 15.262$ (3.162) & -872.296 & Ga: $9.716 \mathrm{e}-01(1.554 \mathrm{e}-01)$ & -869.453 \\
\hline 7 & Fr: $25.157(1.293)$ & -797.564 & $t: \begin{array}{c}9.676 \mathrm{e}-01(8.285 \mathrm{e}-01) \\
12.094(4.334)\end{array}$ & -790.376 \\
\hline 8 & Fr: 22.866 (1.193) & -740.723 & $t: \begin{array}{c}9.622 \mathrm{e}-01(1.726 \mathrm{e}-01) \\
14.159(2.693)\end{array}$ & -727.688 \\
\hline 9 & Fr: $18.971(1.003)$ & -645.247 & $t: \begin{array}{c}9.496 \mathrm{e}-01(1.817 \mathrm{e}-01) \\
11.644(2.537)\end{array}$ & -638.676 \\
\hline 10 & Fr: 18.115 (9.655e-01) & -628.948 & $t: \begin{array}{c}9.446 \mathrm{e}-01(1.598 \mathrm{e}-01) \\
15.031(1.810)\end{array}$ & -625.503 \\
\hline 11 & Fr: $18.914(1.000)$ & -664.500 & $t: \begin{array}{c}9.472 \mathrm{e}-01(1.643 \mathrm{e}-01) \\
12.477(1.236)\end{array}$ & -657.871 \\
\hline 12 & Gu: $4.800(2.347 \mathrm{e}-01)$ & -666.816 & $t: \begin{array}{c}9.435 \mathrm{e}-01(1.562 \mathrm{e}-01) \\
19.326(1.503)\end{array}$ & -647.400 \\
\hline 13 & Ga: 9.371e-01 (1.143e-01) & -662.624 & $t: \begin{array}{c}9.352 \mathrm{e}-01(1.335 \mathrm{e}-01) \\
5.015(3.346 \mathrm{e}-01)\end{array}$ & -649.495 \\
\hline 14 & Ga: 9.062e-01 (9.686e-02) & -544.598 & $t: \begin{array}{c}9.057 \mathrm{e}-01(1.148 \mathrm{e}-01) \\
5.015(3.356 \mathrm{e}-01)\end{array}$ & -537.674 \\
\hline
\end{tabular}

15 t: 9.009e-01 (1.092e-01); 5.012 (3.371e-01) -520.043 Ga: 8.959e-01 (9.219e-02) -514.918

$16 t$ : 8.765e-01 (1.043e-01); 10.513 (1.601) -543.724 Ga: 8.725e-01 (7.888e-02) -537.068

Figures in brackets are standard errors and for Student $t$ copula, the first parameter is correlation and the second one is degree of freedom, " $i$ " denotes the $i^{\text {th }}$ window, "Ga", " $t$ ", "Fr" and "Gu" represent Gaussian, Student $t$, Frank and Gumbel copulas, respectively. 
Table 4

Estimates for the dynamic Student $t$ copula with time-varying degrees of freedom

\begin{tabular}{cllll} 
Subject & static copula & \multicolumn{2}{l}{ time-varying copula } \\
\hline Parameter & $\hat{\rho}$ & $9.349 \mathrm{e}-01(5.095 \mathrm{e}-02)$ & $\hat{\rho}$ & $9.288 \mathrm{e}-01(4.972 \mathrm{e}-02)$ \\
& $\hat{\nu}$ & $5.807(5.078 \mathrm{e}-01)$ & $\hat{s}_{0}$ & $2.551 \mathrm{e}-01(1.314 \mathrm{e}-01)$ \\
& & & $\hat{s}_{1}$ & $-1.574 \mathrm{e}-01(9.108 \mathrm{e}-04)$ \\
& & $\hat{s}_{2}$ & $-5.257 \mathrm{e}-01(2.275 \mathrm{e}-02)$ \\
\hline AIC value & -3797.926 & -3861.514 \\
\hline
\end{tabular}

Figures in brackets are standard errors. 